\title{
Purification of $a_{-1}$-antitrypsin monomer by preparative electrophoresis
}

\author{
F Spada, G Candiano, C Sergi, G M Ghiggeri, F Callea, R Gusmano
}

\begin{abstract}
Alfa-1-antitrypsin ( $\left.a_{1} \mathrm{AT}\right)$ was purified by pseudoligand chromatography and preparative electrophoresis from the serum of a patient with $a_{1} \mathrm{AT}$ deficiency. The combination of the two techniques yielded a high grade batch of $a_{1} \mathrm{AT}$ monomer and this was successfully used to purify the protein from the serum of PiMIM1, PiMIM2, and PizZ phenotype subjects. This procedure should facilitate structural studies of $a_{1} \mathrm{AT}$ variants susceptible to intracellular accumulation.
\end{abstract}

(F Clin Pathol 1994;47:661-663)

Alfa- ${ }_{1}$-antitrypsin $\left(a_{1} \mathrm{AT}\right)$ is a serum glycoprotein with serine protease inhibitor activity. ${ }^{1}$ It is mainly synthesised by the liver where it undergoes specific processing before secretion. In serum $a_{1} \mathrm{AT}$ is microheterogeneous over a narrow range of pIs. The major phenotypes are labelled according to their isoelectric properties, from $M$ (medium mobility) to $\mathrm{Z}$, the most alkaline species. In general, the electrical charge of $\mathrm{PiM}$ species reflects changes in carbohydrate side chains, ${ }^{2}$ but other major differences are due to amino acid substitutions which render $a_{1} \mathrm{AT}$ less soluble and prone to form globular inclusions within hepatocytes. ${ }^{34}$ This was described for both the $\mathrm{Z}$ variant, which presents a $342 \mathrm{Glu} \rightarrow$ Lys substitution, ${ }^{3}$ and for rare $M$-like variants, one of which ( $M$ Cagliari) is characterised by a deletion of 52 Phe. ${ }^{5}$ Technologies for purifying discrete amounts of $a_{1} \mathrm{AT}$ warrant structural studies, including the amino acid sequence of $a_{1} \mathrm{AT}$ pathological variants.

In this paper we describe a two-step

Department of

Nephrology, G Gaslini Institute, Genoa, Italy F Spada

$G$ Candiano

G M Ghiggeri

R Gusmano

1st Department of

Pathology, Spedali

Civili of Brescia, Italy

F Callea

Department of

Pathology, G Gaslini

Institute

C Sergi

Correspondence to:

F Callea, 1st Department of Pathology, Spedali, Civili of Brescia, Brescia 25123, Italy

Accepted for publication

11 January 1994 ciency, and clinical features of chronic liver disease. Blood was collected after an overnight fast and immediately centrifuged; serum was stored at $-20^{\circ} \mathrm{C}$ for less than one month.
The characterisation of the PiZZ molecule was carried out using the monoclonal antibody against PiZZ that is specific only for this mutant ${ }^{6}$; DNA analysis from the same patient was performed according to a method described before. ${ }^{5}$

After 24 hours of dialysis at $4^{\circ} \mathrm{C}$ against $0.03 \mathrm{M}$ phosphate buffer ( $\mathrm{pH} \mathrm{7)} 20 \mathrm{ml}$ of serum was passed through a $16 \times 1.5 \mathrm{~cm}$ column of Affi Gel Blue (BioRad, Richmond, California) equilibrated with the same dialysis buffer. The initial $30 \mathrm{ml}$ volumes were discharged while the following $45 \mathrm{ml}$ were recovered and processed further. In these conditions albumin interacts with the Cibacron Blue F3GA dye, immobilised on agarose via a mechanism which is still unclear, while $a_{1} \mathrm{AT}$ has low affinity for the dye and elutes in the recovered fraction.

Preparative electrophoresis was performed in thick $(2 \mathrm{~mm})$ gels of polyacrylamide $(18 \times 16 \mathrm{~cm})$ without detergents. Gradients of polyacrylamide were cast by mixing two solutions of acrylamide ( $10 \%$ and $18 \%$ ), both containing constant $0.9 \%$ BIS ( $\mathrm{C}=2.5 \%$ ), $40 \%$ sucrose, $0.033 \%$ ammonium persulphate and $0.033 \%$ TEMED in $37 \mathrm{mM}$ TRIS$\mathrm{HCl}$ ( $\mathrm{pH} \mathrm{8.8)}$. The stacking gel was constant in BIS and in acrylamide concentrations (C = $4 \% ; \mathrm{T}=4 \%)$ in $125 \mathrm{mM}$ TRIS- $\mathrm{HCl}(\mathrm{pH}$ 6.8). Electrode buffer was $25 \mathrm{mM}$ TRISglycine $(\mathrm{pH} 8 \cdot 3)$. The sample application point corresponded to the whole length of the upper margin of the gel. The run was continued overnight with an initial 200 volts and constant $60 \mathrm{~mA}$ using a Protean 11 apparatus (Bio Rad) equipped with a $1000-500$ power supply. After electrophoresis the strips relative to $a_{1} \mathrm{AT}$ were cut with a razor blade and the protein, after homogenisation, was recovered with electroelution. Gel strips fragmented in $50 \mathrm{mM}$ TRIS-glycine buffer $(\mathrm{pH}$ 8.9 ) were put into tubes (16 mm in diameter) of a vertical electrophoretic chamber (Bio Rad 175 model tube cell), each tube being closed at the bottom with a plug of polyacrylamide gel of the same composition of the stacking gel. Tubes were connected with a dialysis membrane (Amicon PM 10000 millwatts cutoff) containing $50 \mathrm{mM}$ TRIS-glycine which was immersed in the same buffer and acted as the trapping site. Electroelution was continued overnight by applying a constant 120 Volts corresponding to the initial $80 \mathrm{~mA}$. The final $a_{1} \mathrm{AT}$ concentration was determined using the Coomassie G-250 dye binding assay. 
Figure 1 (a) Separation efficiency of preparative electrophoresis in nondenaturing conditions of an Blue; numbers from 1 to 9 identify the

uncharacterised proteins which were separated by preparative electrophoresis. Immunoblotting with polyclonal antibodies was used to characterise the migrating position in the preparative gels of different then of purified band 8 before (c) and after chromatography on Affi phenotypes migrated into two major bands, one of which (band 8) was easily recovered from the preparative gel. effluent from Affi Gel $a_{1} A T$ phenotypes $(d-e)$ and Gel Blue. All $a_{1} A T$

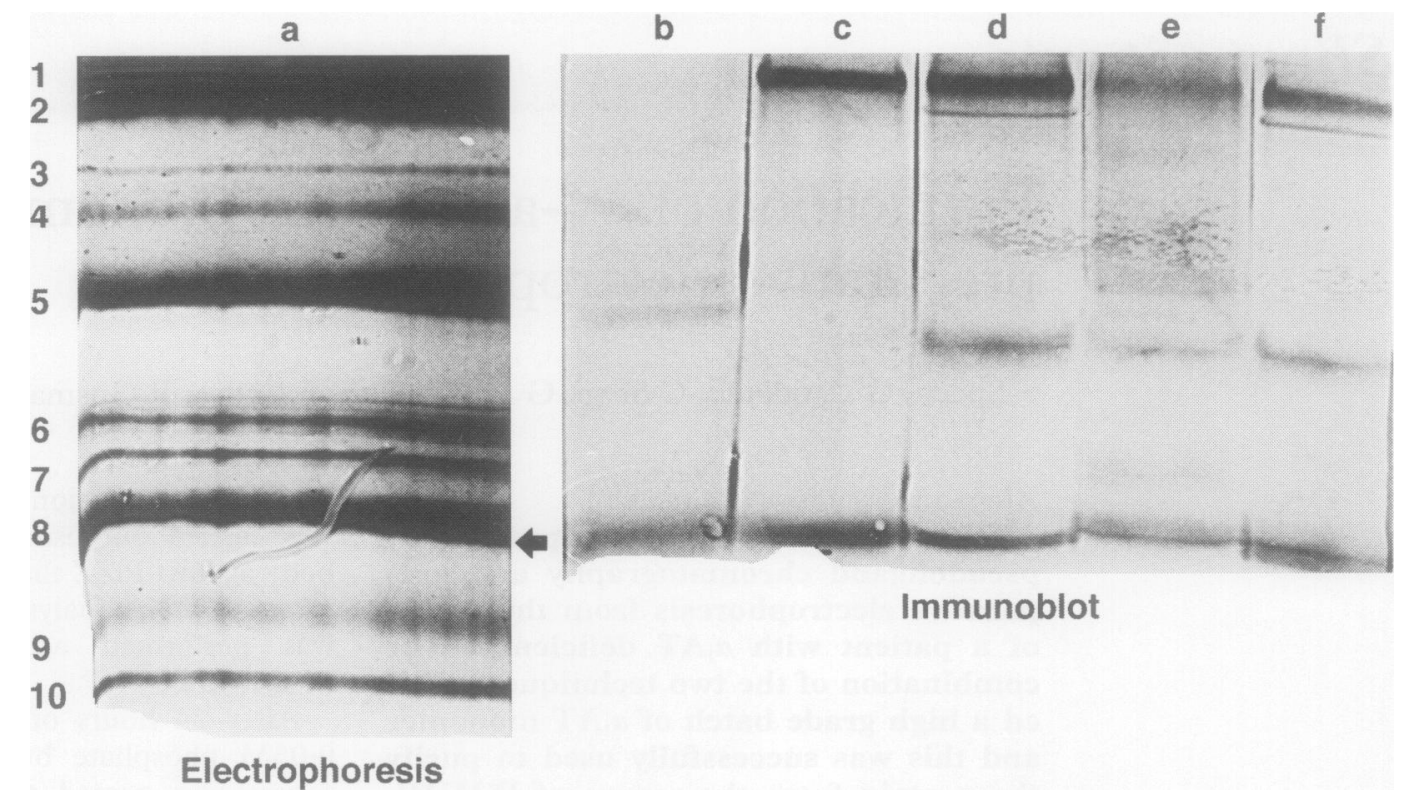

Discontinuous electrophoresis was performed in gradient sodium dodecyl sulphatepolyacrylamide gels $(T=10-20 \% ; C=2.5 \%)$ according to the method of Laemmli. ${ }^{7}$ Gels $(0.7 \mathrm{~mm})$ were cast by mixing two polyacrylamide solutions $(10 \%-20 \%)$ containing $0.9 \%$ BIS, $30 \%$ sucrose, $0.033 \%$ ammonium persulphate and $0.033 \%$ TEMED in $30 \mathrm{mM}$ TRIS-HC1 ( $\mathrm{pH} \mathrm{8 \cdot 8).} \mathrm{The} \mathrm{run} \mathrm{was} \mathrm{continued}$ overnight at an initial 60 Volts (constant 16 $\mathrm{mA}$ ) using the Bio Rad Protean II apparatus.

For immunolocalisation of $a_{1} \mathrm{AT}$, proteins were transblotted to Hybond C-Super (Amersham, Little Chalfont, England) nitrocellulose membranes, using a BioRad dry

\section{b}

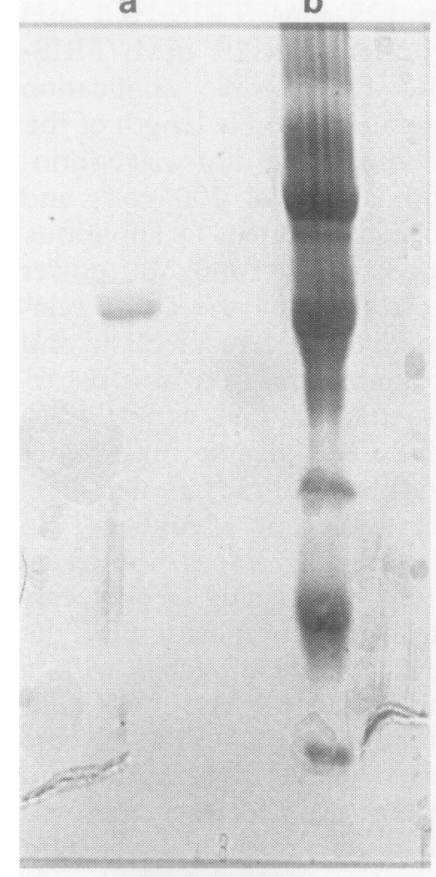

SDS-PAGE c

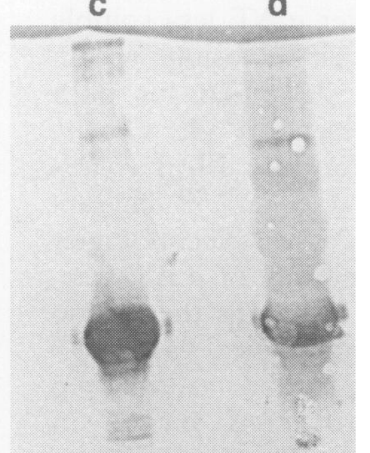

Immunoblot
Figure 2 Analytical SDS-PAGE (a-b) and immunoblotting (c-d) of an effluent from Affi Gel Blue and of purified band 8 from preparative electrophoresis. Band 8 from preparative electrophoresis corresponds to the $a_{1} A T$ monomer. transblot cell. Transfer was carried out for three hours with $300 \mathrm{~mA}$ in $20 \mathrm{mM}$ TRIS, $190 \mathrm{mM}$ glycine (pH 8.3) plus $10 \%$ methanol. Polyclonal antibodies against $a_{1} \mathrm{AT}$ (Dako, Copenhagen, DK) were used for immunoblotting and colour was developed with goat-anti rabbit IgG linked to alkaline phosphatase in the presence of nitroblue tetrazolium and 5-bromo-4-cloro-3-indolylphosphate. Washing steps were done according to the manufacturer's instructions (Bio Rad).

Fused rocket immunoelectrophoresis was carried out in agarose gels using antibodies against $a_{1} \mathrm{AT}$ and against total human serum proteins (Dako)

For sequencing, proteins were transblotted electrophoretically to PVDF membranes in a semi-dry apparatus (Hoefer, San Francisco, California). Sequences were recorded using a Millipore Pro-Sequences 6625 (Bedford, Massachusetts) and the resulting phenylthiohydantoin amino-acid derivatives were identified using the on-line Waters 600 E PTH analyser (Millipore).

Ultrathin isoelectric focusing was performed in polyacrylamide gels containing carrier ampholytes (Pharmacia, Upssala, Sweden) in a narrow range of pIs (pI 4.2$4 \cdot 9)$. Runs were continued for 12 hours at a constant 1200 volts. Proteins were stained with Coomassie R.250.

\section{Results}

The main problem we encountered in purifying $a_{1} \mathrm{AT}$ was the removal of serum albumin, owing to the striking homology in molecular weight and isoelectric point between the two proteins. This was eventually achieved by devising a two-step procedure in which albumin was first immobilised on Affi Gel Blue due to the strong affinity of this protein with the blue dye. Alpha ${ }_{1} \mathrm{AT}$, which does not bind the resin, was instead recovered and further purified using preparative electrophoresis. 
The recovery of $a_{1} \mathrm{AT}$ at this stage was nearly $100 \%$. During preparative electrophoresis without detergents, $a_{1} \mathrm{AT}$ migrated in three different bands (fig 1 , a) one of which (band 8) corresponded to the $a_{1} \mathrm{AT}$ monomer of the purest grade on the basis of the immunoblot (fig 2), and the $\mathrm{NH} 2$ amino terminal sequence. Further analysis of band 8 showed the presence of $\mathrm{M} 1 / \mathrm{M} 2$ phenotypes. This technique was then used to purify serum $a_{1} \mathrm{AT}$ in a case of PiZZ homozygosis. The use of monoclonal anti-Z $a_{1} \mathrm{AT}$ antibody confirmed the $Z$ identity; DNA analysis from the same patient showed a point mutation at the level of the $\mathrm{V}$ exon (GAA-GAG). As already reported for the $M$ species, the analysis of $a_{1} \mathrm{AT}$ composition by immunoblot showed that band 8 accounted for the major part of the $\mathrm{Z}$ variant (fig $2, \mathrm{e}$ ), showing that our method is very effective for purifying this variant. The final recovery of $a_{1} \mathrm{AT}$ was about $50 \%$ in both the case of $M$ and $\mathrm{Z} a_{1} \mathrm{AT}$ species.

\section{Discussion}

Previous techniques for ${ }_{1} \mathrm{AT}$ purification have all been based on serial chromatographic passages or on affinity chromatography. ${ }^{89}$ The one presented here is based on preparative electrophoresis after separation of $a_{1} \mathrm{AT}$ from albumin, which is the most direct contaminant, owing to its strict homology in molecular weight and isoelectric point.

There are at least two major advantages to the newly described method: it allows high grade $a_{1} \mathrm{AT}$ monomer to be purified, thus excluding other components with a different size (dimeric $a_{1} \mathrm{AT}$ or fragmentation products); it works perfectly with both normal and abnormal $a_{1} \mathrm{AT}$ molecules. The demonstration that PiZZ $a_{1} \mathrm{AT}$ can be easily purified electrophoretically makes it a method of choice for structural studies of $a_{1} \mathrm{AT}$ both in vitro and in vivo. It is also simple and highly reproducible.

The highly purified monomers obtained with this method should facilitate physicochemical analysis (crystallography, circular dichromism, amino acid fingerprinting) of the molecule, thus potentially helping to solve the unknowns on polymerisation and the insolubility of all $a_{1} \mathrm{AT}$ variants. ${ }^{10}$

We acknowledge the secretarial help of Mrs Belich. This work was carried out with a grant from the Italian Ministry of Health to the G Gaslini Institute.

1 Beatty K, Bieths J, Travis J. Kinetics of association of serine proteinases with native and oxidized 1-antioproteinase inhibitor and 1-antichymotrypsin. $f$ Biol Chem 1980;255:3931-4.

2 Vaughan L, Carrell R. a1-antitrypsin isoforms: Structural basis of microheteogeneity. Biochem Biophys Res Commun 1981;2:461-7.

3 Lomas DA, Evans DL, Finch J, Carrell R. The mechanism of Z-1-antitrypsin accumulation in the liver. Nature 1992;357:605-7.

4 Jeppsson J, Larsson C, Eriksson S. Characterization of 1antitrypsin in the inclusion bodies from the liver in 1antitrypsin deficiency. N Engl f Med 1975;296:576-9.

5 Sergi C, Consalez GL, Fabbretti G, Brisigotii M, Faa G, Costa V, et al. Immunohistochemical and genetic characterization of the Cagliari AAT molecule (M. Cagliari acterization of the Cagliari AAT molecule
AAT Deficiency) Lab Invest 1994;70:130-3.

6 Callea F, Brisigotti M, Faa G, Lucini L, Eriksson S. Identification of $\mathrm{PiZ}$ gene products in liver tissue by a monoclonal antibody specific for the $\mathrm{Z}$ mutant of 1 antitrypsin. F Hepatol 1991;12:372-6.

7 Laemmli UK. Cleavage of structural proteins during the assembly of the head of bacteriophage T4. Nature 1970;227:680-5.

8 Murthy R, Hercz A. Purification of human a1-antitrypsin by affinity chromatography on sepharose bound concanavalin-A. FEBS Letts 1973;32:243-6.

9 Musiani P, Tomasi T. Isolation, chemical and physical properties of al-antitrypsin. Biochemistry 1976; properties

10 Sifers RW. $\mathrm{Z}$ and the insoluble answer. Nature 1992;357:541-2. 